\title{
Energy Harvesting in Wireless Sensor Networks: Techniques and Issues
}

\author{
Kishor Singh and Sangman Moh \\ Dept. of Computer Eng., Chosun Univ., Gwangju, Korea \\ ikishor_singh@yahoo.com,smmoh@chosun.ac.kr
}

\begin{abstract}
Wireless sensor networks (WSNs) have been an immensely effective and inseparable part of wireless technology in a plethora of applications and thus succeeded in captivating the attention of research world for more efficient performance. Often times, WSNs need to be deployed over the regions where consistent monitoring, supervision and maintenance turn out to be a herculean task. Under such scenarios, WSNs could not be exploited to their full potential; one of the major hurdles is the limited battery power that is unable to meet the long-term energy requirement. As a result, it is focused on to convert ambient energy into electric form. Even though battery technology and low-power devices are evolving, the changes are relatively slow and do not meet the energy requirement yet. Energy harvesting, conversion of ambient energy into electrical energy, has therefore emerged as an effective alternative to powering the sensor networks. This paper reviews the most preferred ambient sources available for energy harvesting and the scavenging techniques commonly used in WSNs to deal with energy issues. Furthermore, the energy harvesting techniques are qualitatively compared, and the challenges and open issues in the design and implementation of energy harvesters are discussed in depth.
\end{abstract}

Keywords: Wireless sensor network, energy consumption, energy harvesting, energy conversion, network lifetime

\section{Introduction}

With the unprecedented development of microelectronics, integrated circuits, and wireless communications in the recent years, wireless sensor networks (WSNs) have emerged as a promising technology. A WSN consists of spatially distributed, inexpensive miniaturized devices called sensors which are deployed over a geographical area for the close monitoring of physical conditions [1]. Information can be gathered by measuring environment variables such as temperature, pressure, humidity, sound, motion, etc.

The sensor nodes are enabled with sensing, computing and communicating capabilities to ensure that they can sense the target attributes, process them, and transmit the collected data to the designated center or sink. Besides, they tend to communicate with one another through wireless channels to accomplish the exchange of information. All the processes consume significant amount of the energy stored in capacity-limited batteries. It is, therefore, not possible for WSNs with limited power capabilities to afford long-term operation without any external or supplementary energy source [2]. The energy consumption in a typical WSN mainly depends on following aspects:

- Battery: discharging rate, battery size, the electrode used, charging time, current leakages, load current.

- Sensors: sensing, signal conversion, sampling frequency, signal conditioning, analog to digital conversion.

- Radio transceivers: transmission power, reception power, ranges, duty cycle, and modulation technique. 
- Processor: processing speed, operating frequency, processor power, and ambient temperature.

Sensor nodes are likely to operate only for a finite duration of time. The network lifetime gets even more aggravated when nodes are placed in remote and hostile places such that battery replacement becomes almost impossible and the storage of sufficiently enough energy is out of question. Energy, thus, appears as the most pressing constraint that can restrict the lifetime of a WSN as a whole. In the scarcity of energy, once the battery gets dead, the sensor node simply becomes functionless. Such scenarios are critically severe in the applications such as battlefields or habitat monitoring. Using a large-capacity battery to enhance the lifetime can be thought of, but this will have to trade off with increased weight, size and cost as well. The lifetime of sensor nodes can also be prolonged by compromising with the performance parameters [3]. For example, the use of a low-power processor or low-power transmitter can slow down the depletion of battery energy but it has to deal with lower processing speed and smaller transmission ranges, adding up net delay.

Several endeavors have been made towards energy efficient routing, duty cycling strategies, power control mechanism, energy-aware MAC protocols, data aggregation, and data compression with an objective of conserving energy. All the techniques do contribute in improving energy utilization but have nothing to add to the limited battery capacity. The fact that battery technology is getting better and better and the power consumption of electronic devices is lowering with the passage of time cannot be denied. But, this has not been able to keep the pace with the exponentially growing applications of WSNs which stress on long life, small size, low cost, less weight, and minimal environment impact. This has prompted the relevant researchers to shift their interest to the development of technologies that could meet the increasing energy needs perpetually and supplement the on-board battery with cost effectiveness. Eventually, the idea of extracting energy from the surrounding resources, energy harvesting, came into existence and has proved its worthiness in providing uninterrupted power supply and dealing with energy constraints.

The sources of energy in our natural environment exist in many forms, are distributed widely, and have endless supply which could be exploited indisputably [4]. More importantly, they are readily available in abundance without requiring any extra effort to produce them. A variety of ambient sources has been used for energy scavenging, which are solar energy, mechanical energy from vibration, stress and strain, thermal energy from heating sources like furnaces, electromagnetic energy induced from inductors, coils and transformers, RF energy from the radio signals, and wind energy from the wind.

Energy harvesting from ambient resources stands as reliable and effective means for empowering the small autonomous sensor nodes. Energy harvesting not only lessen the energy dearth but also is more likely to free the node dependency on power-limited batteries. A WSN capable of energy harvesting enables long-term operation and improves not only network performance but also communication reliability. In addition, the harnessed electrical energy contributes to optimizing the performance of the network without any kind of trade-off. The nodes can increase their sampling frequency or duty cycle for more accuracy whereas the radio transceivers can increase their transmission range and power to quicken the routing process. All of the attributes can be collectively achieved only if the sensor network is bestowed with abundant energy.

The implementation of energy harvesting technologies is not simple. A large number of things need to be taken into account for effective energy conversion and efficient use. The nature and characteristics of different energy sources happen to be entirely different. Hence, the techniques and transducers vary in each case. In most cases, the ambient energy resources may not be consistent and provide energy in small packets. So, capturing and storing the energy packets are never easy and require a permanently active harvesting circuit. Such harvesting circuit needs to be small, robust and energy-efficient. Note that the energy consumption in the harvesting circuit must be very small compared to the 
energy provided by the ambient sources. The rate of energy harvesting should always exceed the rate at which the energy is consumed. Because the energy harvesting is not possible all the times, it is always a challenge for the harvested energy to be maintained at stable supply voltage. That is, a reliable and durable storage system is necessarily required.

The rest of the paper is organized as follows: The components of an energy harvesting system integrated to sensor nodes are overviewed in the following section. The commonly used energy harvesting techniques and the ambient energy sources with associated techniques are presented in Section 3. In Section 4, the energy harvesting techniques are qualitatively compared and technically discussed. In Section 5, important challenges and open issues are addressed. The paper is concluded in Section 6.

\section{Energy Harvesting System}

Energy harvesting is the technique of converting the unused ambient energy source into electrical energy. The energy obtained is called harvested energy. The energy harvesting basically consists of three major components: namely, energy source, energy harvesting architecture and energy consuming entity [5]. An energy harvesting system captures and scavenges the ambient energy and then supplies the harvested and converted electrical energy to sensor nodes. The design of the energy harvesting system may differ from one application to another depending on the type of load requirements and the conditions of deployment area [6]. The design and implementation of an efficient energy harvesting system not only requires proper selection of devices with appropriate ratings but also the proper integration of those electronics to the sensor node. Figure 1 shows a usual energy harvesting system integrated with a sensor node.

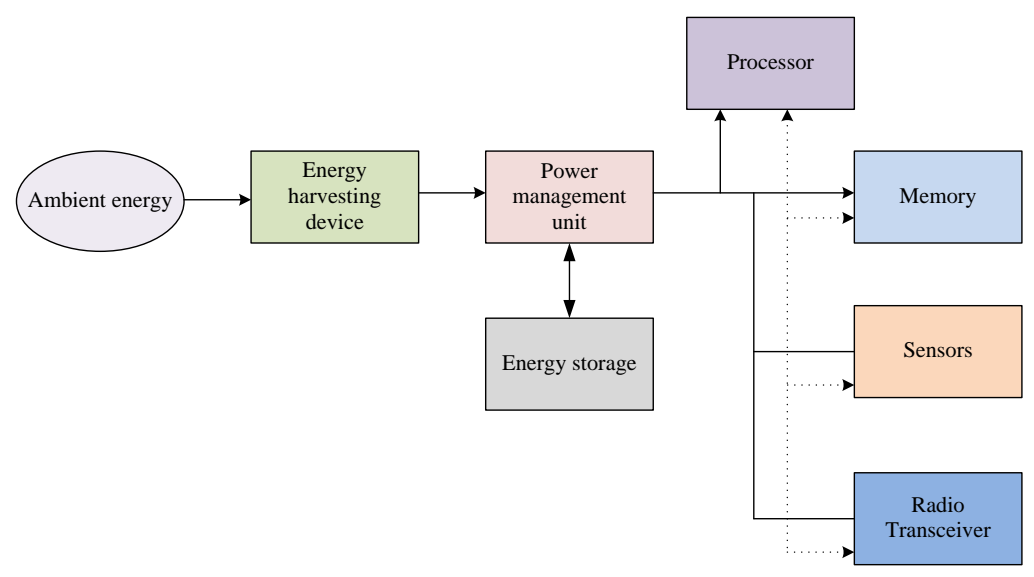

Figure 1. Energy Harvesting System

In the schematic of Figure 1, the source of energy called ambient energy refers to all energy in our surrounding such as solar energy, wind energy, thermal energy, and mechanical energy. The beauty of energy sources is their renewal property with limitless availability. Most of them are safe and clean energies with no harmful effect on system and environment. They, however, exhibit varied characteristics in terms of controllability, predictability, availability, and magnitude. Each energy source has different harvesting capabilities which accounts for varying harvester design for each type and eventually leads to different energy conversion efficiencies [7].

The energy harvesting device in Figure1 simply refers to the device or conversion mechanism adopted for transforming harnessed ambient energy into electrical energy. They are a form of transducers. Some of the most commonly used energy harvesting devices include solar cell, wind turbine generator, piezoelectric film or ceramic, thermocouples, and pyroelectric materials. The selection of such devices is very tricky 
indeed because the performance of an energy harvesting device depends on how well the device delivers energy. It is, therefore, necessary to consider various factors such as the nature and characteristics of ambient energy source, the type of energy storage used, the target application, and most importantly the size, cost and power consumption of the device. It is always advantageous if the harvesting device can provide ample power that is quite enough for the sensor node at an appropriate voltage.

The power management unit also called power conditioning unit is a crucial part of the energy harvesting system. Energy management becomes mandatory to ensure continuous and consistent energy supply because the energy harvesting is comparatively random and intermittent process. The major function of this unit is to draw energy from the harvesting device, manages the storage of energy with proper conditioning, and delivers the regulated power to the load. The output voltage of the harvesting device is mostly discontinuous in nature whereas the sensors are highly sensitive to the change in voltage. Therefore, a need of voltage regulating body arises to deliver a stable and regulated voltage to the sensor node. Likewise, it is required to control the charging voltage for energy storage and to ensure efficient consumption of stored energy. And care must be given to ensure that conversion devices are precisely scaled and calibrated.

Even though the harvested energy can be directly used in the sensor network, storing the extra energy is always an added advantage. Whenever the ambient energy sources become temporarily unavailable, the stored energy enables continued load operation. This is what emphasizes the need of energy storage devices either in the form of rechargeable batteries or capacitors. In most of the cases, a battery is used as energy storage because it has high energy density and better discharging rates. Also, the rechargeable batteries come with different technologies and battery chemistry like Lithium-ion, Nickel-cadmium, and Nickel-metal-hydride. Batteries, however, suffer from problems such as limited recharge cycles, current leakage, smaller operation life, etc. Supercapacitors, on the other hand, have high power density, and longer lifetime than batteries. At the same time, they offer lower impedance, rapid charging and discharging, and virtually unlimited charging cycles [8]. The concerns of heat dissipation and overcharging do not remain with the use of ultracapacitors and they do not release anything causing environmental degradation.

Obviously, all the component of a sensor node needs energy invariably for their basic functioning and operations. As referred to Figure1, the main energy consuming components are processor, sensor, radio transceiver, and memory. The important point to be noted here is that optimal power supply improves the performance of each of these components. Again, energy is inevitable for WSNs but is a serious constraint.

\section{Energy Harvesting Techniques}

Environmental energy sources have been used to generate electricity for a long time. The governing principle of energy conversion is basically the same even in WSNs. There are, however, complex tradeoffs to be considered when designing energy harvesting circuits for WSNs, arising from the interaction of various factors such as the characteristics of the energy sources, the energy storage device used, the power management functionality of the nodes, the protocols used, and the requirements of applications. This is why different energy harvesting techniques implement different approaches for harnessing the ambient energy. In the following subsections, we discuss the energy harvesting techniques which are commonly used in WSNs.

\subsection{Photovoltaic Energy Harvesting}

Photovoltaic energy harvesting is the technique of converting solar energy into electrical energy. In other words, photons are converted into moving electrons. This process is based on photoelectric effect. A combination of solar cell and rechargeable batteries is good enough to provide the node with eternal lifetime. Solar is an abundant 
energy resource with limitless availability. It is one of the most efficient ambient energy sources with highest power density, and it is perfect for outdoor network applications. Moreover, solar is a green energy and causes no harmful effect to the environment, which is the reason behind its use in energy harvesting purpose for a long time.

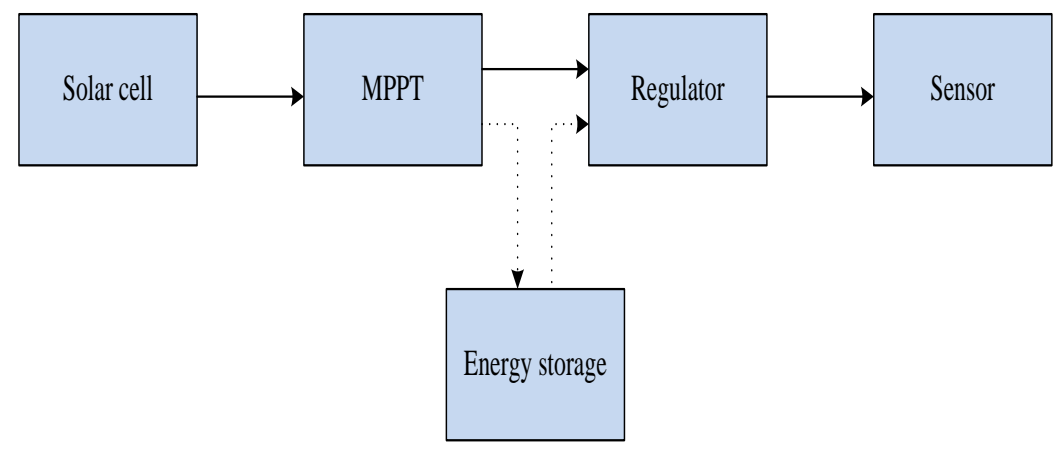

Figure 2. Solar Energy Harvesting System

Figure 2 depicts a general working model of the solar energy harvesting system for WSNs. The significance of the system lies in solar cell, maximum power point tracker (MPPT), and voltage converter. A solar cell converts the solar energy into the electrical energy. In general, solar cells come in a lot of varieties like monocrystalline, polycrystalline and amorphous cells. It must be chosen as per our requirement. Typically, the monocrystalline cells have the highest efficiency. MPPT makes sure that the maximum power is extracted from the solar cell by matching the impedance of the solar cell and the voltage regulator or converter [9]. The output characteristics of the photovoltaic system vary nonlinearly with the changing irradiance and time of the day. Under such scenario, MPPT automatically track the value of current and voltage at which maximum output power is delivered.

In solar energy harvesting systems, batteries are usually used to store surplus energy for night or time of low solar irradiance. But it is not uncommon to find the use of supercapacitors for storage purpose. A combination of a set of ultracapacitors in parallel connection with a series of alkaline batteries is used in [10] as energy storage module. A voltage regulator can be used for the purpose of maintaining the required stable voltage for the sensor node. Regulated voltage is of much importance to sensor nodes that are otherwise sensitive to changing voltages. The major advantage with solar energy harvesting is easy installation process, reliability, and safety from electrical hazards. Nevertheless, solar is uncontrollable energy resource and its availability is unpredictable, resulting in new harvesting challenges for reliable and efficient power management. Also, the energy conversion efficiency drops significantly in indoor applications. The solar energy harvesting can be applied in both grid-connected and off-grid power system in diverse applications like powering traffic lights, repeater stations, satellites, and space stations.

\subsection{Wind Energy Harvesting}

Wind is a commonly available ambient energy resource with a tendency of making WSN completely self-autonomous with sustainable energy. Wind energy harvesting is particularly attractive because of the ubiquitous availability of wind power and its relatively high power density. An added advantage with wind energy harvesting systems is that necessary wind can be generated locally with devices like electrical fan. 


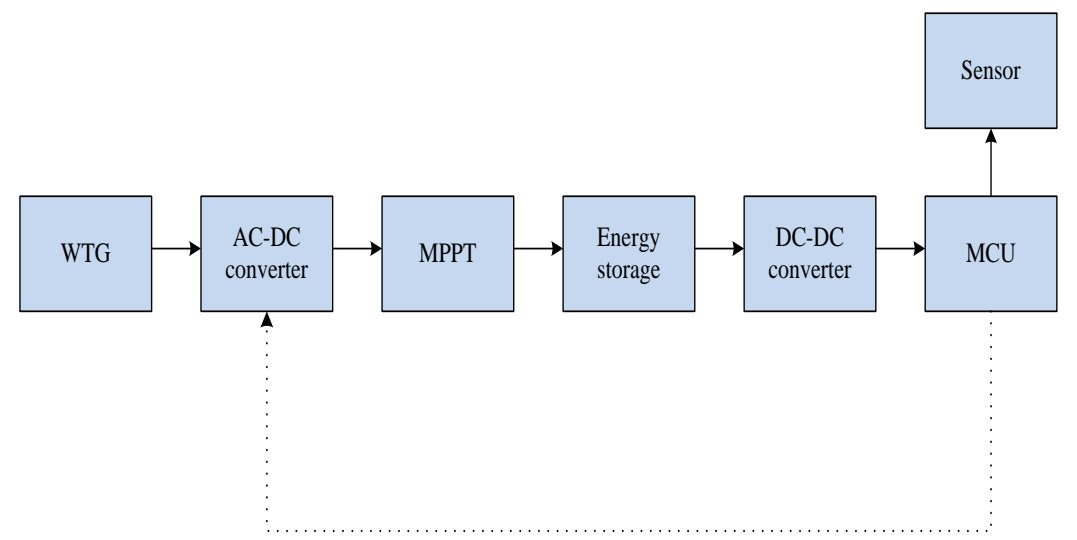

Figure 3. Wind Energy Harvesting System

Figure 3 shows a schematic diagram of a typical wind energy harvesting system. In [11], a wind turbine generator (WTG) is used as the energy harvesting device, consisting of a wind turbine coupled to an electrical generator. This combination allows the conversion of kinetic energy of air flow into mechanical energy by the turbine, followed by conversion of mechanical energy of turbine into electrical energy with the electrical generator. The latter is based on the simple process of electromagnetic induction. The current generated is alternating or direct depending upon the type of generator. The power output of a WTG depends on the velocity of the air, contact area, and its efficiency. An alternating current (AC) generator is used along with a rectifier to convert the induced $\mathrm{AC}$ into direct current (DC). A semiconductor-based rectifier is used to improve the conversion efficiency.

In [12], a DC generator has been used, and it simply requires DC-DC converter in-stead of the rectifier. The use of MPPT ensures extraction of maximum power from WTG. A low-power microcontroller (MCU) implements the algorithm for MPPT. For the purpose of energy storage, battery or supercapacitor or both can be used. A supercapacitor is used as it has virtually unlimited life cycles and fast charging/discharging mechanisms. The DC-DC converter regulates the level of voltages appropriate for sensors and is controlled by MCU.

A properly designed wind energy harvesting system can provide perpetual energy supply such that sensor nodes never run out of energy. It opens up the door for using WSNs in the most severe and hostile conditions. Nevertheless, there are some bottlenecks in its implementation. First, the system is generally large-sized. To get them into a smaller size, it costs a lot. Because of mechanical parts involved, the system can be a bit noisy and constantly varying wind strength can make the process troublesome. The stochastic nature of wind not only hinders the energy harvesting process but also imbalances demand and supply. In addition, miniaturization is another major issue with the wind energy system where the challenge of designing turbines in proportion to WSN network exists.

\subsection{Piezoelectric Energy Harvesting}

The general working mechanism of piezoelectric energy harvesting system is shown in Figure 4. Piezoelectric devices convert mechanical force, stress or vibration into electrical energy. In other words, they produce electric polarization when strained mechanically and vice versa. For example, the industry-based WSNs can be easily power from the vibrations of floors and walls caused by nearby machinery through harvesting techniques. It is based on the phenomenon called piezoelectric effect which is the ability to convert the deformation caused by stress into electric potential difference. Typically, a vibrationbased energy harvester constitutes a mass-spring system that resonates when subjected to 
vibrations from the surroundings. The low-power vibrations are thereafter amplified to enhance output power. In fact, a higher output power is possible only when the system is in resonance with the external exciting vibration [13]. The use of piezoelectric harvesters is getting popular for the fact that they need no external voltage, possesses high electromechanical coupling ability, and are well fitted for microelectromechanical systems. Piezoelectric materials are receiving a great deal of attention because of their rugged and simple structure, ease of implementation, ubiquitous availability, no electromagnetic interference, low cost, and most importantly high efficiency. Energy harvesting is the technique of converting solar energy into electrical energy. In other words, photons are converted into moving electrons. This process is based on photoelectric effect. A combination of solar cell and rechargeable batteries is good enough to provide the node with eternal lifetime. Solar is an abundant energy resource with limitless availability. It is one of the most efficient ambient energy sources with highest power density, and it is perfect for outdoor network applications. Moreover, solar is a green energy and causes no harmful effect to the environment, which is the reason behind its use in energy harvesting purpose for a long time.

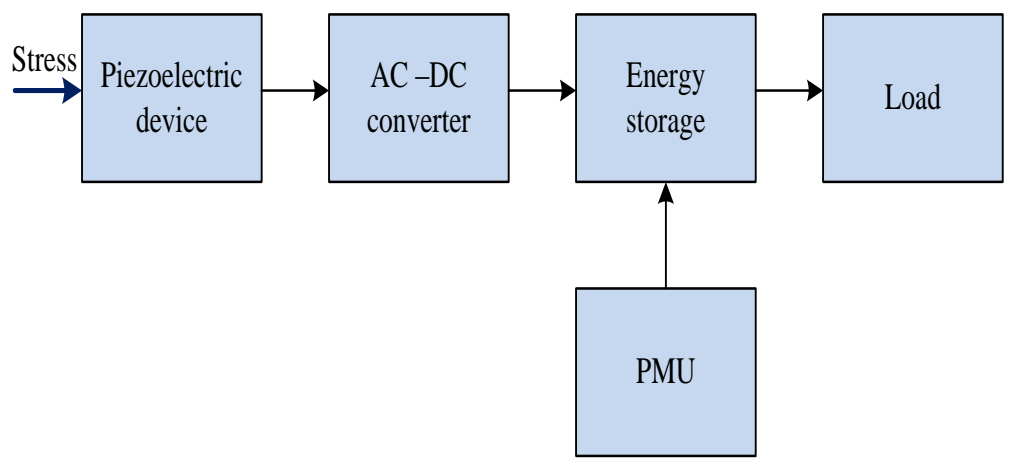

Figure 4. Piezoelectric Energy Harvesting System

The piezoelectric energy harvesting system consists of a piezoelectric material bonded to a cantilever beam [14]. When the beam gets excited by some ambient vibration, a significant strain develops in the piezoelectric patch which is transformed into electrical energy. Lead-Zirconate-Titanate (PZT) is mostly used piezoelectric ceramic for vibrationto-electrical energy conversion. The AC voltage generated during the process is converted and regulated by the rectifying circuit for storage. The amount of electrical energy generated during the process depends on the frequency and amplitude of the vibration.

In [15], a trapezoidal piezoelectric harvester has been used for harnessing electrical energy from ambient vibrations. The device has an excitation frequency of $100 \mathrm{~Hz}$ and the resonance is achieved with a software operated trapezoidal cantilever. To achieve higher strains, a mass can be added to the free end of the cantilever. The main advantage of this material is that it shows no deterioration or fatigue even for cycles of the order $10^{7}$. They can be easily integrated into a system, resulting in their potential of perfect energy harvesting source for growing energy applications. Moreover, vibration is one of the most prevalent energy with its availability in different forms in buildings, roads, bridges, vehicles, and many others. There are, however, certain problems with most of the piezoelectric material including the brittleness and current leakage. For an instance, piezoelectric energy harvesting system with cantilever beam, which is the mostly used form of energy harvesting of this type, cannot withstand a wide range of exciting vibration. 


\subsection{RF Energy Harvesting}

A radio frequency (RF) energy harvesting system allows the energy-constrained WSNs to harvest energy from the radio signals for performing their operations. Ambient RF signals obtained from cellular mobile communication systems, televisions (TVs), and wireless local area networks (WLANs) can be harvested to power sensor nodes. The radio signal can be used to carry energy in the form of electromagnetic radiation to a system in the far field. With the concept RF energy harvesting, the simultaneous transmission of information and energy has become possible. A sensor node can use a single radio both for RF harvesting and for communicating with others nodes in a WSN and thus contributes to reducing the complexity of the network.

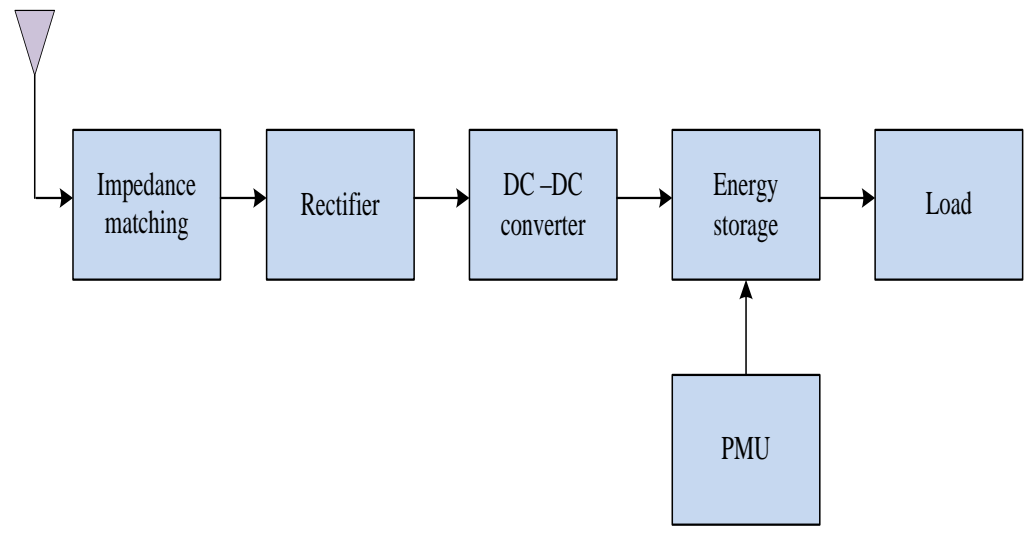

Figure 5. RF Energy Harvesting System

A typical RF energy harvesting is shown in Figure 5, where the main component is called RF energy harvester or rectenna. It is a combination of impedance matching, rectifier, and DC-DC converter, and it is devised to collect radio signals and convert them to corresponding electrical equivalent with maximum efficiency. The use of Schottky diode in the rectifier can improve the efficiency of power conversion significantly [16]. The highest efficiency is achieved when the rectifier impedance is matched to the antenna impedance. The power management unit (PMU) acts as a buffer between the rectenna and the energy storage device. It makes sure that energy is stored with no loss.

In [17], far-field RF energy harvesting has been implemented to energize a low-power and low-duty-cycle WSN with radio signals in gigahertz band. Data transmission, which is the most energy consuming operation, is not performed continuously. Hence, harnessed energy is stored during the periods of no data transmission. A closed loop monitoring system is implemented to accomplish adaptive adjustment of data transmission duty cycle according to the level of energy stored.

Unlike other energy sources such as solar and wind, RF sources deliver more controlled and constant energy. Also, RF energy harvesting does not depend on nature, and thus it provides relatively predictable energy supply. In fact, the amount of the harvested RF energy depends on the wavelength of the harvested RF signal and the distance between an RF energy source and the harvesting device which can be de-signed as per need. The transmit power level, however, must be restricted within the limits recommended by international standards to avoid potential health problems. Note that the regulations regarding the health and safety should always be taken care of. If designed and implemented properly, it can be used for indoor and outdoor applications including agricultural monitoring, smart homes, structural health monitoring, object detection and location tracking. 


\subsection{Thermal Energy Harvesting}

Thermal energy harvesting is another topic of interest for the researchers working in the field of energy harvesting. It is based on the simple principle of converting heat into the electrical energy. One of the most commonly used methods for achieving this mechanism is see-back effect, where the temperature difference across two end joints of a pair of dissimilar metals accounts for electrical current. More importantly, the amount of the current produced is proportional to the temperature difference. A specific application of thermoelectric energy harvesting would be monitoring the health of patient utilizing the heat from the patient's body.

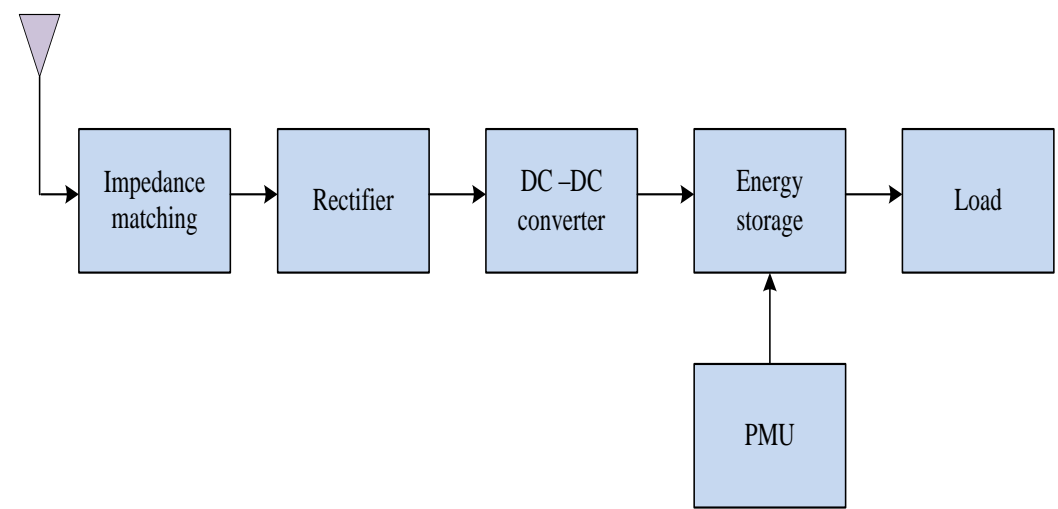

\section{Figure 6. Thermal Energy Harvesting System}

A thermal energy harvesting system is shown in Figure 6, the inevitable component of which is a thermoelectric generator (TEG). TEG basically consists of a pair of p-type and n-type semiconductor connected in series electrically but in parallel thermally between two ceramic layers [18]. With TEG, thermal gradients in the environment can be easily and effectively converted into electrical energy for powering sensor nodes. A well designed DC-DC converter is used to boost the low voltages at the harvester output and thus enhance the system efficiency at the end. The PMU ensures the efficient utilization of harvested energy by regulating the power distribution across the network and energy storage as well.

A thermal powered WSN is presented in [19], with a simplified power manager (PM). The PM balances the performance of the system by changing the duty cycle of the node such that the energy is in proportion the harvested energy. Supercapacitor is used as a main storage element, the voltage level of which determines the active or sleep period of the sensor node.

The scavenging process in thermal energy harvesting is comparatively easy. It is suitable for both indoor and outdoor applications. Nevertheless, thermal energy harvesting systems have low efficiency and limited capability of harvesting energy even when the temperature gradient is around $30^{\circ} \mathrm{C}$ to $40^{\circ} \mathrm{C}$, making it more suitable for low-power applications. Therefore, considerable research efforts have been made to come up with alternative methods to garner thermal energy. In this regard, the development of microthermoelectric generator and thermally-sensitive ion generation mechanism would be highly productive.

\section{Comparison of Energy Harvesting Techniques}

The most common forms of energy harvesting techniques have been reviewed and thereby compared qualitatively and presented in Table.1. The simple objective of all these energy harvesting schemes is to generate appropriate and sufficient energy for the sensor nodes to operate and store the excess energy for the future purpose. 
Solar, though, is a matured and established technology in renewable energy harvesting, developing the same in small scale for sensitive networks like WSNs involves challenges because the output power is directly proportional to exposed surface area. Invention of micro solar cells and implementation of MPPT technology has significantly improved the efficiency of solar based energy harvesting systems. Under the most usual condition the efficiency is found to be varying between $10-25 \%$. An array of solar cells connected $n$ series and parallel is the usual mode of scavenging solar energy. The major advantage with solar energy lies in the fact that it is green energy with limitless availability. However, the point to be noted that solar based system are functional only when get exposure to the sunlight. The efficiency drops if the intensity of the light downfalls and in areas, devoid of light, especially the indoor applications the performance of the photovoltaic systems is low.

Wind is another ambient energy source often used for the purpose of powering wireless sensor networks. Wind Turbine Generator (WTG) is usual energy harvester used; however, it comes out to be sophisticated and expensive when miniaturized for WSN purpose. The implementation of MPPT technique is a must to adapt wind varying conditions and achieve maximum efficiency. Likewise, with a combination of efficient rectification and power conditioning WEH can surely be used to power sensor networks perpetually with significant improvement in overall energy conversion. Nevertheless, wind being uncontrollable and unpredictable needs special caring and techniques to get the best out of it. Usually, wind based energy scavenging system consist of mechanical parts which may produce unnecessary noise. This might prove a serious setback for sensor networks to be applied for sensitive applications.

Piezoelectric energy harvesting is the most appropriate form of energy harnessing mechanism for fabricated environment. The amount of energy produced depends on the magnitude of the stress applied and the frequency of the vibration generated. So, it is more or less dependent on the environment or apparatus set for excitation or generating vibration. Also it is determined by the nature of the piezoelectric devices, especially how they react to the deformation. The beauty of piezoelectric materials is the unchanged efficiency they deliver in both indoor and outdoor applications. Unfortunately, the most commonly used piezoelectric substance like PZT are brittle in nature; hence they may not perform to the longest time.

RF energy harvesting being the wireless energy transfer puts an added advantage over the wired or connected system. The development and implementation of RF based system needs to consider the characteristics of propagation channel, distance from the RF transmitter, design of the rectenna, antenna size and transmit power. The problem with this system is the significant fading with RF energy with increasing distance. On top, the transmit power of radio signals need to be restricted within the limits set by the international standards and may vary with countries. The use of dedicated radio source, however, can improve the efficiency over ambient radio signal which are low powered otherwise.

Thermal energy harvesting, on the other hand, is inexpensive method of converting the temperature gradient into electrical energy. For an efficient thermal energy harvesting system, the design of efficient TEG and heating system has to be taken into account. Maintaining a significant temperature gradient across TEG with an ideal combination of heat exchanger to meet heat requirements and heat sink for cooling effect is needed. Since the thermal energy systems are more prone to charge leakage, care must be taken to prevent the energy loss. 
Table 1. Comparison of Different Energy Harvesting Techniques for Wsns

\begin{tabular}{|c|c|c|c|c|c|}
\hline $\begin{array}{c}\text { Harvesting } \\
\text { Technique }\end{array}$ & $\begin{array}{c}\text { Energy } \\
\text { Source }\end{array}$ & Energy Type & $\begin{array}{c}\text { Harvesting } \\
\text { Device }\end{array}$ & Merit & Demerit \\
\hline $\begin{array}{c}\text { Solar energy } \\
\text { harvesting [9] }\end{array}$ & Sun & Radiant energy & Solar cell & $\begin{array}{c}\text { Limitless } \\
\text { availability }\end{array}$ & $\begin{array}{c}\text { Poor indoor } \\
\text { efficiency }\end{array}$ \\
\hline $\begin{array}{c}\text { Wind energy } \\
\text { harvesting [11] }\end{array}$ & Wind & Kinetic energy & Wind turbine & $\begin{array}{c}\text { High power } \\
\text { density }\end{array}$ & $\begin{array}{c}\text { Noisy } \\
\text { Unpredictable }\end{array}$ \\
\hline $\begin{array}{c}\text { Piezoelectric } \\
\text { energy har- } \\
\text { vesting [13] }\end{array}$ & $\begin{array}{c}\text { Stress or } \\
\text { vibration }\end{array}$ & $\begin{array}{c}\text { Mechanical } \\
\text { energy }\end{array}$ & $\begin{array}{c}\text { Piezoelectric } \\
\text { film or } \\
\text { ceramic }\end{array}$ & $\begin{array}{c}\text { Equally } \\
\text { efficient } \\
\text { indoor and } \\
\text { outdoor }\end{array}$ & $\begin{array}{c}\text { Piezoelectric } \\
\text { devices are } \\
\text { brittle }\end{array}$ \\
\hline $\begin{array}{c}\text { RF energy } \\
\text { harvesting [16] }\end{array}$ & $\begin{array}{c}\text { Radio } \\
\text { signals }\end{array}$ & $\begin{array}{c}\text { Electromagnetic } \\
\text { energy }\end{array}$ & $\begin{array}{c}\text { Rectenna } \\
\text { mochanical } \\
\text { parts }\end{array}$ & $\begin{array}{c}\text { Fading with } \\
\text { distance }\end{array}$ \\
\hline $\begin{array}{c}\text { Thermal energy } \\
\text { harvesting [18] }\end{array}$ & Heat & Heat energy & $\begin{array}{c}\text { Thermoelectric } \\
\text { generator }\end{array}$ & $\begin{array}{c}\text { Easy and } \\
\text { simple }\end{array}$ & $\begin{array}{c}\text { Current } \\
\text { leakage }\end{array}$ \\
\hline
\end{tabular}

\section{Challenges and Open Issues}

Integrating energy harvesting systems to WSNs requires a lot of processes that involves capturing, storing, conditioning, regulating, and managing. Furthermore, it is hugely tough to take into consideration all these factors collectively. The miniaturization of the energy harvesting system to fit the requirements of WSNs is always a challenging job. Followed are the challenges and open issues in this regard.

\subsection{Conversion Efficiency}

The power conversion efficiency of an energy harvester and the overall system is always an issue of interest. For most of the harvesting techniques, the overall conversion efficiency turns out to be low. The major reason is the significant power consumed by semiconductor devices and power elements which are involved in the development of harvesting devices such as converters, voltage boosters, rectifiers, etc. Therefore, great research efforts and precise experimental observations are necessarily needed for designing and developing fundamental materials, fabrication technologies, and integration methods. The multidisciplinary approach of natural sciences and engineering can really contribute to the efficiency improvement of energy harvesting systems.

\subsection{Power Management}

In addition to harvesting power from the ambient sources, it is also important that the harnessed power is efficiently stabilized, stored and delivered to the targeted destinations. The energy provided by the harvester is usually discontinuous in nature and unstable. But, since the WSN nodes can operate only with stabilized DC, designing an efficient rectifier or DC-DC converter is of huge significance. At the same time, the output voltage of harvesting systems is not high enough. So the need of DC-DC boosters with more efficient MPPT algorithm is required. The power management unit should not only improve the efficiency of harvesting system but also make sure that it consumes a minimal amount of energy. Further research and development of interfacing circuits are also crucial. 


\subsection{Energy Storage}

The excess energy obtained from the harvesting system need to be stored efficiently. Because the energy supplied by harvesters is discontinuous and intermittent, the task of generating a continuous and constant supply goes to energy storing devices. Although batteries have high energy density, the problem regarding their disposal, self-discharging, and limited life cycles needs to be addressed. Supercapacitors can be used to meet longterm applications as they have infinite charging and discharging cycles. But, they suffer from large leakage current along with low energy density. Therefore, the design of appropriate and efficient storage mechanism is always challenging. A combination of battery and capacitor can be am alternative. As a result, researches are required to delve further not only to assess the tradeoffs between the battery and capacitor but also their possible combination.

\subsection{Topology Control}

The control over the transmit power and regulation of convenient wake-up and sleep schedules for sensor nodes can help in efficient conservation of hard-earned energy. The analysis of incoming traffic patterns can be used for determining the sleep time for nodes on the basis of transmission delays. Care must also be given to the battery state, applications being served, and environmental conditions to deter-mine the periods for sleep mode. Moreover, this technique requires precise synchronization among the member nodes to make sure that transmission and reception get accomplished with no unnecessary idle listening.

\subsection{Energy-Aware Protocols}

Energy harvesting in itself is a great effort to address the energy constraint in WSNs. Implementing the harvesting technique is just not enough to reap all the bene-fits of energy-harvested WSNs. In fact, the governing protocols for different layers including routing at the network layer and MAC at the data link layer must comply with the harvesting environment. The usual communication protocols for WSNs may not deliver their best and are much likely to degrade the performance of the network when applied directly. Therefore, it is much important that the protocols are adapted to leverage the availability of energy harvesting in the network. In summary, in addition to the development efforts of the energy harvesting systems, there exists a big room for improvement in the domain of developing a suitable protocol stack for energy-harvesting WSNs.

\section{Conclusion}

One major factor that restricts the diverse and long-term applications in WSNs is the energy-constrained power supply. Recently, various energy harvesting techniques to provide perpetual power supply have been developed and implemented for prolonging the lifetime of WSNs. In this paper, the most widely used and efficient techniques for energy harvesting in WSNs have been extensively reviewed and qualitatively compared. Undoubtedly, the recent advancement of the energy harvesting systems is offering a huge supplement to the conventional battery-dependent systems. With the unprecedented development of efficient microelectronics and relevant technologies, the power consumption of sensor nodes is gradually decreasing. Hence, it is most likely that the harvesting technologies will prove their worth in making WSNs completely independent of battery power. The challenges and important issues on the way to more efficient harvesting are also discussed in this paper. The need for smart grid technologies to deal with variability would be an effective solution for extracting energy in a most efficient 
way. To sum up, energy harvesting with the ambient energy resources is reliable, safe, and clean, and it has the potential to power WSNs endlessly.

\section{Acknowledgements}

This paper is a revised and expanded version of a paper entitled "A Comparative Survey of Energy Harvesting Techniques for Wireless Sensor Networks" presented at the 3rd International Conference on Green and Smart Technology, Jeju, Korea, Dec. 21-23, 2016 [20]. This research was supported in part by Basic Science Research Program through the National Research Foundation of Korea (NRF) funded by the Ministry of Education (NRF-2016R1D1A1A09918974). Correspondence should be addressed to Dr. Sangman Moh (smmoh@chosun.ac.kr).

\section{References}

[1] I. Akyildiz, W. Su, Y. Sankarasubramaniam, and E. Cayirci, "Wireless sensor networks: A survey", Computer Networks, vol. 38, no. 4, (2002), pp. 393-422.

[2] S. Sudevalayam and P. Kulkarni, "Energy Harvesting Sensor Nodes: Survey and Implications", IEEE communications surveys \& tutorials, vol. 13, no. 3, (2011), pp. 443-461.

[3] S. Basagni, M. Y. Naderi, C. Petrioli, and D. Spenza, "Wireless sensor networks with energy harvesting", Edited S. Basagni, M. Conti, S. Giordano, and I. Stojmenovic, Mobile Ad Hoc Networking: Cutting Edge Directions, John Wiley \& Sons, Inc., Hoboken, NJ, (2013), pp. 703-736

[4] G. Zhou, L. Huang, W. Li, and Z. Zhu, "Harvesting ambient environmental energy for wireless sensor networks: a survey", Journal of Sensors, (2014), pp. 1-20.

[5] A. S. M. Z. Kausar, A. W. Reza, M. U. Saleh and H. Ramiah, "Energizing wireless sensor networks by energy harvesting systems: Scopes, challenges and approaches", Renewable and Sustainable Energy Reviews, vol. 38, (2014), pp. 973-989.

[6] Y. K. Tan and S. K. Panda, "Review of energy harvesting technologies for sustainable WSN", Edited W. Seah and Y. K. Tan, Sustainable Wireless Sensor Networks, Vellore, India: InTech., (2010), pp. 15-43.

[7] W. K. G. Seah, Z. A. Eu, and H. P. Tan, "Wireless sensor networks powered by ambient energy harvesting (WSN-HEAP) - survey and challenges", Wireless VITAE, (2009), pp. 1-5.

[8] F. K. Shaikh and S. Zeadally, "Energy harvesting in wireless sensor networks: A comprehensive review", Renewable and Sustainable Energy Reviews, vol. 55, (2016), pp. 1041-1054.

[9] J. Gakkestad and L. Hanssen, "Powering Wireless Sensor Networks Nodes in Northern Europe Using Solar Panel for Energy Harvesting," Proceedings of 4th IFIP International Conference on New Technology, Mobility and Security (NTMS), Paris, France, (2011), pp.1-5.

[10] K. V. Naveen and S. S. Manjunath, "A reliable ultracapacitor based solar energy harvesting system for Wireless Sensor network enabled intelligent buildings", Proceedings of $2^{\text {nd }}$ International Conference on Intelligent Agent \& Multi-Agent Systems, Chennai, India, (2011), pp. 20-25.

[11] Y. K. Tan, and S. K. Panda, "Optimized wind energy harvesting system using resistance emulator and active rectifier for wireless sensor nodes", IEEE Transaction on Power Electronics, vol. 26, no. 1, (2011), pp. 38-50.

[12] Y. Wu, W. Liu, and Y. Zhu, "Design of a wind energy harvesting wireless sensor node", Proceedings of $3^{\text {rd }}$ International Conference on Information Science and Technology (ICIST), (2013), pp. 1494-1497.

[13] Y. J. Yoon, W. T. Park, K. H. Li, Y. Q. Ng and Y. Song, "A study of piezoelectric harvesters for lowlevel vibrations in wireless sensor networks", International Journal of Precision Engineering and Manufacturing, vol. 14, no. 7, (2013), pp.1257-1262.

[14] A. Nechibvute, A. Chawanda, and P. Luhanga, "Piezoelectric energy harvesting devices: an alternative energy source for wireless sensors", Smart Materials Research, (2012), pp. 1-13.

[15] E. K. Reilly, F. Burghardt, R. Fain, and P. K. Wright, "Powering a wireless sensor node with a vibration-driven piezoelectric energy harvester", Smart Materials and Structures, vol. 20, no. 12, (2011), pp. 125006.

[16] H. J. Visser and R. J. M. Vullers, "RF energy harvesting and transport for wireless sensor network applications: Principles and requirements", Proceedings of the IEEE, vol. 101, no. 6, (2013), pp. 14101423.

[17] Z. Popovic, E. A. Falkenstein, D. Costinett, and R. Zane, "Low-power far-field wireless powering for wireless sensors", Proceedings of the IEEE, vol. 101, no. 6, (2013), pp. 1397-1409.

[18] X. Lu and S.-H. Yang, "Thermal energy harvesting for WSNs", Proceedings of IEEE International Conference on Systems Man and Cybernetics (SMC), (2010), pp. 3045 -3052.

[19] T. N. Le, A. Pegatoquet, O. Sentieys, O. Berder, and C. Belleudy, "Duty Cycle Power Manager for Thermal-Powered Wireless Sensor Networks," Proceedings of 24th IEEE International Symposium on Personal, Indoor and Mobile Radio Communication (PIMRC), London, UK, (2013), pp. 144-149. 
[20] K. Singh and S. Moh, "A Comparative Survey of Energy Harvesting Techniques for Wireless Sensor Networks", Proceedings of 3rd International Conference on Green and Smart Technology (GST), Jeju, Korea, (2016), pp. 28-33.

\section{Authors}

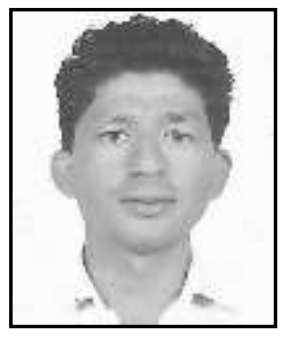

Kishor Singh, received the B.E. degree in electronics and communication from Khwopa Engineering College, Purbanchal University, Nepal in 2013 and is pursuing M.S. degree in computer engineering at Chosun University, Korea. His current research interests include cognitive radio networks with a focus on network architectures and relevant protocols.

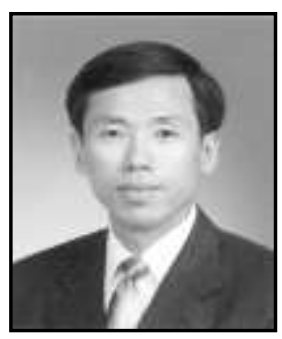

Sangman Moh, received the Ph.D. degree in computer engineering from Korea Advanced Institute of Science and Technology (KAIST), Korea in 2002. Since late 2002, he has been a professor in the Department of Computer Engineering at Chosun University, Korea. From 2006 to 2007, he was on leave at Cleveland State University, USA. Until 2002, he had been with Electronics and Telecommunications Research Institute (ETRI), Korea, where he served as a project leader, since he received the M.S. degree in computer science from Yonsei University, Korea in 1991. His research interests include mobile computing and networking, ad hoc and sensor networks, cognitive radio networks, and parallel and distributed computing systems. He has published more than 200 papers in international and domestic journals and conference proceedings, and has held more than 40 overseas and domestic patents. He serves on the program committees of international conferences and workshops in his areas of interest. Dr. Moh is a member of the IEEE, the ACM, the IEICE, the KIISE, the IEIE, the KIPS, the KICS, the KMMS, the IEMEK, the KISM, and the KPEA. 